\title{
Platelet to lymphocyte ratio is associated with the severity of coronary artery disease and clinical outcomes of percutaneous coronary intervention in the Chinese Han population
}

\author{
DONG ZHOU ${ }^{1,2}$, GUANGYAO WANG $^{3}$, YAN FAN ${ }^{2}$, ZHAOFEI WAN ${ }^{2}$ and XIAOJUN LIU ${ }^{2}$ \\ ${ }^{1}$ Department of Cardiovascular Medicine, Hanzhong Central Hospital, Hanzhong, Shaanxi 723000; \\ ${ }^{2}$ Department of Cardiovascular Medicine, First Affiliated Hospital of Medical School, Xi'an \\ Jiaotong University, Xi'an, Shaanxi 710061; ${ }^{3}$ Department of Cardiovascular Medicine, Guangxi \\ Zhuang Autonomous Region Brain Hospital, Liuzhou, Guangxi 545005, P.R. China
}

Received June 19, 2015; Accepted August 23, 2016

DOI: $10.3892 /$ etm.2016.3993

\begin{abstract}
The aim of the present study was to evaluate whether the ratio of the absolute number of platelets to the number of lymphocytes (PLR) correlates with the severity of coronary artery disease (CAD) and major adverse cardiovascular disease (CVD) events in Chinese patients with CAD. PLR was calculated as follows: PLR=platelet count/lymphocyte count, using the complete blood counts of 854 Chinese Han subjects. CAD severity was determined using angiographic evidence by cardiologists unaware of the study aims. The association between PLR and CAD severity was analyzed by logistic regression. Clinical endpoints were evaluated during a median follow-up period of 42 months. The association between PLR and CVD events was assessed using Cox regression models. Patients with PLR $>171$ exhibited more severe coronary artery stenosis [odds ratio, 2.393; 95\% confidence intervals (CI), 1.394-4.108; $\mathrm{P}=0.002$ ] and worse prognoses, with a higher rate of major adverse CVD events during five years of follow-up (hazard ratio, 1.982; 95\% CI, 1.329-2.957; $\mathrm{P}=0.001$ ). A Kaplan-Meier curve demonstrated that the CVD event rate of $34.27 \%$ in patients with PLR $>171$ was significantly higher than that in patients with $\mathrm{PLR}<100(\mathrm{P}<0.001)$. These findings suggest that PLR is independently associated with CAD severity and long-term major adverse CVD events; therefore, high PLR may predict poor prognosis of CAD in the Chinese Han population.
\end{abstract}

Correspondence to: Dr Dong Zhou, Department of Cardiovascular Medicine, Hanzhong Central Hospital, 22 Kangfu Road, Hanzhong, Shaanxi 723000, P.R. China

E-mail: zhoudong8587@126.com

Professor Guangyao Wang, Department of Cardiovascular Medicine, Guangxi Zhuang Autonomous Region Brain hospital, 1 Jila Road, Liuzhou, Guangxi 545005, P.R. China

E-mail: 494183396@qq.com

Key words: coronary artery disease, platelet, lymphocyte, ratio

\section{Introduction}

Coronary artery disease (CAD), which is characterized pathologically by atherosclerosis, is a predominant cause of death in developed countries. The chronic inflammatory response is a critical element in the pathogenesis of atherosclerosis (1), which is associated with the production of platelets and lymphocytes. Elevated platelet counts accelerate atherosclerosis formation, progression, and destabilization of atherosclerotic plaques, and are significantly associated with long-term incidence of fatal CAD in apparently healthy individuals (2). Platelets can act as a bridge between local inflammation at the vascular wall and the development of atherosclerotic plaques (3). Platelets are activated by substances released from cells of the vascular wall and secrete proinflammatory chemokines and cytokines associated with vascular inflammation (4). Activated platelets promote the development of atherothrombotic disease or eventually result in cardiovascular disease (CVD) events (5). In addition, lymphocyte deficiency may also accelerate the progression of atherosclerosis (6). In addition to predicting long-term recurrent myocardial infarction (MI) in patients with ST-segment elevation MI, a low lymphocyte count may also predict the risk of reduced hemodynamics and aerobic capacity in patients with heart failure (7). A low lymphocyte count is also an independent predictor of poor outcomes for patients with heart failure during hospitalization (8).

Therefore, the ratio of the absolute number of platelets to the number of lymphocytes [platelet/lymphocyte ratio (PLR)] may act as an effective biomarker to predict CVD and the risk of future CVD events (9). Although counts of leukocytes and their subsets have been demonstrated to be associated with the severity of CAD (10), the relationship between PLR and CAD severity remains unclear. Furthermore, the majority of previous studies have focused on western patients (11-13). There are numerous differences between Western and Chinese populations, including environment and genetics, which may explain the differences observed in platelet counts and white blood cell counts between the two populations (14). The role of PLR in predicting the risk of CVD events in Chinese patients following percutaneous coronary intervention (PCI) remains uncertain. 
The aim of the present study was to evaluate whether PLR correlates with the severity of CAD and CVD events in Chinese patients with CAD.

\section{Materials and methods}

Ethics statement. The present study was approved by the Ethics Committee of the First Affiliated Hospital of Xi'an Jiaotong University (Xi'an, Shaanxi, China), and was performed in accordance with the guidelines of the Declaration of Helsinki. All participants were provided with the research information and granted written, informed consent.

Study protocol and patient selection. In the first experimental stage, 854 Chinese Han subjects (523 males; 331 females), who were admitted to the Department of Cardiology at the First Affiliated Hospital of Xi'an Jiaotong University, were recruited between January 2009 and July 2013. All subjects in the age range of 24-88 years old underwent coronary angiography. CAD severity was defined as the number of major epicardial coronary vessels with angiographic evidence of one or more stenoses of $\geq 50 \%$ severity (15). The method of evaluation was described previously by Arbel et al (16). Based on the findings of coronary angiography, recruited subjects were classified into the control ( 0 -vessel; $n=215)$, 1-vessel CAD ( $n=328)$, 2-vessel CAD $(n=213)$ or 3-vessel CAD ( $n=229)$ groups.

In the second experimental stage, the relationship between PLR and CVD events in Chinese patients with CAD was evaluated. This prospective cohort study included 639 patients with CAD (age, 31-82 years) who underwent PCI between January 2009 and July 2013 in the Department of Cardiology at the First Affiliated Hospital of Xi'an Jiaotong University, due to serious coronary artery stenosis. According to the tertiles of baseline PLR level, patients with CAD were divided into three groups (tertile 1, <101.00; tertile 2, 101.00-171.00; tertile $3,>171.00$ ). There were 213 patients in each tertile group. Subjects underwent follow-up from 14 to 60 months (median follow-up duration, 42 months) to evaluate the incidence of CVD events. Patients were excluded from both stages of the study if they currently or previously experienced acute infections, acute stroke, advanced liver disease, renal failure, malignancies or blood diseases.

Demographic and clinical data. Investigators gathered the clinical data from all subjects via a standard structured questionnaire. A history of smoking was defined as either past or current consumption of cigarettes. Hypertension was defined as a systolic or diastolic blood pressure $>140$ or $>90 \mathrm{mmHg}$, respectively. Diabetes mellitus (DM) was defined as an elevated fasting plasma glucose concentration $(>126 \mathrm{mg} / \mathrm{dl})$, or serum glycosylated hemoglobin A1C levels $>6.5 \%$. Body weight $(\mathrm{kg})$ and height $(\mathrm{m})$ measurements were acquired from each patient during the initial visit. Body mass index (BMI) was calculated as follows: Body weight $(\mathrm{kg}) /$ height squared $\left(\mathrm{m}^{2}\right)$.

Coronary angiography and PCI. Coronary angiographies and PCI were performed according to the Judkins technique (17). Coronary angiography results were assessed by two independent cardiologists, blinded to the study design, to determine the grade of the coronary stenosis. Severity of coronary artery disease was assessed using Gensini scores (18). In the prospective cohort, patients with CAD underwent target vessel revascularization and implantation of a coronary stent in the coronary arteries with $>70 \%$ luminal narrowing.

Determination of PLR and laboratory measurements. Blood samples $(5 \mathrm{ml})$ were collected into tubes containing anticoagulant following admission. Complete blood count and subgroup number analysis results were obtained within $60 \mathrm{~min}$ following collection of the samples at the hospital laboratory using an XT-4000 automated hematology analyzer (Sysmex Corp., Kobe, Japan). PLR was calculated from the equation: PLR= platelet counts/lymphocyte counts. In addition, the concentrations of uric acid, high sensitivity C-reactive protein (hs-CRP), fasting glucose, triglyceride, total cholesterol and high density lipoprotein cholesterol (HDL-C) were measured. Low-density lipoprotein cholesterol was calculated using the Friedewald formula (19).

Definition of CVD events and follow-up of the cohort patients treated with PCI.CVD events include cardiovascular mortality, nonfatal MI, unstable angina, stroke and revascularization procedures. In the present cohort, 639 patients underwent PCI and were followed-up at average intervals of 1, 3, 6, 12, 24, 36, 48 and 60 months following discharge via telephone communication or face-to-face interviews with patients or their family members. Complete clinical endpoints were obtained from all patients in the cohort upon follow-up. CVD event incidence in the different PLR groups were calculated.

Statistical analysis. Continuous variables were presented as the mean \pm standard deviation. Categorical variables were presented as frequencies and percentages. The Kolmogorov-Smirnov test was used to assess the normality of data distribution. One-way analysis of variance was performed to compare multiple groups. Differences between two groups were compared using the Student's t-test for continuous variables, and the $\chi^{2}$ test or Fisher's exact test for categorical variables, as appropriate. Multinomial logistic regression with normal coronaries was used as the reference category and three blocks were used to evaluate the influence of different risk factors on CAD severity. Evaluation was performed as previously described (16). Cox proportional hazards model analyses were performed to determine independent factors associated with cardiovascular events. Kaplan-Meier survival curves were constructed to further evaluate the prognostic value of PLR. Hazard ratios (HRs) were presented with $95 \%$ confidence intervals (CIs) to demonstrate the risk of an event when this factor was present. All computations were performed with SPSS software 17.0 (SPSS Inc., Chicago, IL, USA). P $<0.05$ was considered to indicate a statistically significant difference.

\section{Results}

Clinical characteristics of the study population. Baseline data for each group are presented in Table I. No significant differences were observed between 0 -vessel control subjects and 1-vessel, 2-vessel or 3-vessel subjects with respect to age, gender and smoking status. The prevalence of risk factors for 
Table I. Clinical characteristics of the study population grouped by the number of diseased vessels.

\begin{tabular}{|c|c|c|c|c|c|c|c|}
\hline Characteristic & 0 -vessel & 1-vessel & P-value & 2-vessel & P-value & 3-vessel & P-value \\
\hline Total, n & 215 & 197 & & 213 & & 229 & \\
\hline Age, year & $58.52 \pm 11.08$ & $58.72 \pm 13.25$ & 0.872 & $59.77 \pm 11.08$ & 0.244 & $60.12 \pm 9.76$ & 0.105 \\
\hline Male, n (\%) & $130(60.47)$ & $114(57.87)$ & 0.617 & $122(57.28)$ & 0.556 & $157(68.56)$ & 0.091 \\
\hline Smoking, n (\%) & $103(47.91)$ & $85(43.15)$ & 0.373 & $108(50.70)$ & 0.629 & $129(56.33)$ & 0.087 \\
\hline $\mathrm{HT}, \mathrm{n}(\%)$ & $56(26.05)$ & 65 (32.99) & 0.131 & $91(42.72)$ & $<0.001$ & $123(53.71)$ & $<0.001$ \\
\hline DM,n (\%) & $18(8.37)$ & $19(9.64)$ & 0.731 & $32(15.0)$ & 0.036 & 48 (20.96) & $<0.001$ \\
\hline $\mathrm{BMI}, \mathrm{kg} / \mathrm{m}^{2}$ & $24.56 \pm 2.56$ & $24.27 \pm 3.05$ & 0.300 & $24.71 \pm 2.63$ & 0.567 & $24.90 \pm 2.90$ & 0.194 \\
\hline WBC, k/ul & $6.78 \pm 2.35$ & $7.35 \pm 1.92$ & 0.007 & $7.22 \pm 2.14$ & 0.047 & $7.07 \pm 1.98$ & 0.168 \\
\hline Lymphocyte, k/ul & $1.99 \pm 0.80$ & $1.93 \pm 0.81$ & 0.479 & $1.82 \pm 0.66$ & 0.021 & $1.70 \pm 1.05$ & 0.001 \\
\hline PLT, k/ul & $211.24 \pm 50.37$ & $217.83 \pm 45.88$ & 0.167 & $221.23 \pm 64.25$ & 0.074 & $224.72 \pm 53.42$ & 0.007 \\
\hline PLR & $116.98 \pm 35.61$ & $127.71 \pm 42.93$ & 0.006 & $140.22 \pm 65.90$ & $<0.001$ & $170.04 \pm 80.88$ & $<0.001$ \\
\hline $\mathrm{TC}, \mathrm{mmol} / \mathrm{l}$ & $3.99 \pm 0.79$ & $4.05 \pm 1.00$ & 0.543 & $4.13 \pm 1.05$ & 0.137 & $4.13 \pm 1.17$ & 0.161 \\
\hline $\mathrm{TG}, \mathrm{mmol} / \mathrm{l}$ & $1.68 \pm 0.66$ & $1.63 \pm 0.78$ & 0.487 & $1.77 \pm 0.80$ & 0.197 & $1.78 \pm 0.87$ & 0.143 \\
\hline HDL-C, mmol/l & $1.03 \pm 0.22$ & $1.04 \pm 0.25$ & 0.888 & $1.00 \pm 0.20$ & 0.086 & $0.99 \pm 0.23$ & 0.088 \\
\hline LDL-C, mmol/1 & $2.35 \pm 0.66$ & $2.36 \pm 0.79$ & 0.931 & $2.40 \pm 0.71$ & 0.425 & $2.47 \pm 1.04$ & 0.144 \\
\hline Hs-CRP, mg/l & $2.09 \pm 0.79$ & $2.15 \pm 0.87$ & 0.409 & $2.43 \pm 0.90$ & $<0.001$ & $2.82 \pm 1.42$ & $<0.001$ \\
\hline Statin, n (\%) & $187(86.98)$ & $179(90.86)$ & 0.273 & $198(92.96)$ & 0.053 & $210(91.70)$ & 0.123 \\
\hline ACEI/ARB, n (\%) & $90(41.86)$ & $91(46.19)$ & 0.427 & $107(50.23)$ & 0.099 & $116(52.97)$ & 0.071 \\
\hline$\beta$-blocker, n (\%) & $131(60.93)$ & $182(92.39)$ & $<0.001$ & $194(91.08)$ & $<0.001$ & $212(92.14)$ & $<0.001$ \\
\hline Aspirin, n (\%) & $190(88.37)$ & $178(90.36)$ & 0.312 & $195(91.55)$ & 0.335 & $212(92.58)$ & 0.088 \\
\hline
\end{tabular}

Data are presented as the mean \pm standard deviation. Significant differences $(\mathrm{P}<0.05)$ are indicated in bold. Vessel, number of major epicardial coronary vessels with angiographic evidence of one or more stenosis; HT, hypertension; DM, diabetes mellitus; BMI, body mass index; WBC, white blood cell count; PLT, platelet; PLR, platelet to lymphocyte ratio; TC, total cholesterol; TG, triglyceride; HDL-C, high-density lipoprotein cholesterol; LDL-C, low-density lipoprotein cholesterol; Hs-CRP, high-sensitivity c-reactive protein; ACEI, angiotensin converting enzyme inhibitor; ARB, angiotensin receptor antagonist.

Table II. Comparison of the severity of coronary artery disease in different tertiles of PLR.

\begin{tabular}{|c|c|c|c|c|c|c|}
\hline & 1-vessel & & 2-vessel & & 3-vessel & \\
\hline PLR & OR $(95 \% \mathrm{CI})$ & P-value & OR $(95 \% \mathrm{CI})$ & P-value & OR $(95 \% \mathrm{CI})$ & P-value \\
\hline PLR $<101$ & 1.000 & & 1.000 & & 1.000 & \\
\hline PLR 101-171 & $1.437(0.884-2.335)$ & 0.136 & $1.850(1.147-2.984)$ & 0.012 & $1.998(1.199-3.329)$ & 0.008 \\
\hline PLR $>171$ & $1.773(1.090-2.882)$ & 0.021 & $1.975(1.188-3.285)$ & 0.009 & $2.393(1.394-4.108)$ & 0.002 \\
\hline
\end{tabular}

Significant differences $(\mathrm{P}<0.05)$ are indicated in bold. PLR, platelet to lymphocyte ratio; CI, confidence intervals; OR, odds ratio; vessel, number of major epicardial coronary vessels with angiographic evidence of one or more stenoses.

CAD, such as DM and hypertension, was significantly higher in the 3-vessel group than in the 0 -vessel group $(\mathrm{P}<0.001)$. Notably, the lymphocyte counts decreased whereas the platelet counts and PLR increased with the increasing number of coronary artery stenoses (Table I).

Association between PLR and CAD severity. To compare the value of PLR with that of other recognized cardiovascular risk factors, a multistep binary logistic regression analysis was performed. The adjusted odds ratio (AOR) from logistic regression analysis was calculated with respect to age, gender, hypertension, DM, smoking status, HDL-C and TC. As shown in Table II, the AOR for 2-vessel CAD was 1.850 (95\% CI, 1.147-2.920; $\mathrm{P}=0.014)$ for PLR between 101 and 171, whereas the higher PLR group demonstrated a higher AOR of 1.975 (PLR $>171$; 95\% CI, 1.188-3.285; $\mathrm{P}=0.009$ ), both of which were statistically significant. Furthermore, the AOR for 3-vessel CAD was 1.998 (95\% CI, 1.199-3.329; P=0.008) for PLR between 101 and 171, and the higher PLR group again demonstrated a higher AOR (2.393; PLR>171; 95\% CI, 1.394-4.108; $\mathrm{P}=0.002$ ), both of which were statistically significant. These findings indicated that PLR was associated with CAD severity and there was a positive correlation between higher PLR values and CAD severity. 
Table III. Clinical characteristics of patients grouped by tertiles of PLR at baseline.

\begin{tabular}{|c|c|c|c|c|}
\hline Characteristic & Tertile $1(n=213)$ & Tertile $2(n=213)$ & Tertile $3(n=213)$ & P-value \\
\hline Age, year & $58.79 \pm 11.75$ & $59.73 \pm 11.38$ & $60.18 \pm 10.85$ & 0.434 \\
\hline Male, n (\%) & $127(59.62)$ & $135(63.38)$ & $131(61.50)$ & 0.728 \\
\hline Smoking, n (\%) & $95(44.60)$ & $105(49.30)$ & $117(54.93)$ & 0.102 \\
\hline HT, n (\%) & $97(45.54)$ & $91(42.72)$ & $91(42.72)$ & 0.795 \\
\hline DM, n (\%) & $29(13.62)$ & $31(14.55)$ & $39(18.31)$ & 0.366 \\
\hline History of MI, n (\%) & 33 (15.49) & $38(17.84)$ & $36(16.90)$ & 0.808 \\
\hline $\mathrm{BMI}, \mathrm{kg} / \mathrm{m}^{2}$ & $24.43 \pm 2.94$ & $24.43 \pm 2.89$ & $25.07 \pm 2.75$ & 0.028 \\
\hline WBC, k/ul & $7.25 \pm 1.54$ & $7.27 \pm 2.06$ & $7.57 \pm 2.29$ & 0.191 \\
\hline Lymphocyte, k/ul & $2.69 \pm 0.70$ & $1.60 \pm 0.46$ & $1.14 \pm 0.37$ & $<0.001$ \\
\hline PLT, k/ul & $202.28 \pm 49.10$ & $222.28 \pm 51.36$ & $242.50 \pm 56.13$ & $<0.001$ \\
\hline $\mathrm{TC}, \mathrm{mmol} / \mathrm{l}$ & $4.02 \pm 1.00$ & $4.14 \pm 1.01$ & $4.16 \pm 1.22$ & 0.377 \\
\hline $\mathrm{TG}, \mathrm{mmol} / \mathrm{l}$ & $1.73 \pm 0.80$ & $1.68 \pm 0.69$ & $1.78 \pm 0.96$ & 0.517 \\
\hline HDL-C, mmol/1 & $1.03 \pm 0.21$ & $1.01 \pm 0.24$ & $0.99 \pm 0.24$ & 0.324 \\
\hline LDL-C, mmol/l & $2.38 \pm 0.79$ & $2.44 \pm 0.97$ & $2.42 \pm 0.82$ & 0.806 \\
\hline Hs-CRP, mg/l & $2.23 \pm 0.97$ & $2.50 \pm 1.21$ & $2.72 \pm 1.16$ & $<0.001$ \\
\hline Gensini score & $52.37 \pm 30.43$ & $64.43 \pm 41.32$ & $77.53 \pm 46.40$ & $<0.001$ \\
\hline Statin, n (\%) & $196(92.02)$ & $197(92.49)$ & $194(91.08)$ & 0.864 \\
\hline ACEI/ARB, n (\%) & $108(50.70)$ & $105(49.30)$ & $111(52.11)$ & 0.957 \\
\hline$\beta$-blocker, n (\%) & $196(92.02)$ & 197 (92.49) & $194(91.08)$ & 0.846 \\
\hline Aspirin, n (\%) & $196(92.02)$ & 195 (91.55) & $194(91.08)$ & 0.941 \\
\hline
\end{tabular}

Data are presented as the mean \pm standard deviation. Significant differences $(\mathrm{P}<0.05)$ are indicated in bold. HT, hypertension; DM, diabetes mellitus; MI, myocardial infarction; BMI, body mass index; WBC, white blood cell count; PLT, platelet; TC, total cholesterol; TG, triglyceride; HDL-C, high-density lipoprotein cholesterol; LDL-C, low-density lipoprotein cholesterol; Hs-CRP, high-sensitivity c-reactive protein; ACEI, angiotensin converting enzyme inhibitor; $\mathrm{ARB}$, angiotensin receptor antagonist.

Comparison of clinical characteristics among CAD patients. To evaluate the association between PLR and clinical outcomes, follow-up was performed with 639 patients with CAD. These patients were divided into three groups according to the tertiles of baseline PLR levels (tertile 1, <101.00; tertile 2, 101.00-171.00; tertile 3, >171.00). Primary baseline characteristics of the prospective cohort study groups are presented in Table III. Significant differences were observed in Gensini score $(\mathrm{P}<0.001)$, BMI $(\mathrm{P}=0.028)$ and hs-CRP $(\mathrm{P}<0.001)$ among three groups. However, there were no significant differences in the other clinical characteristics among the groups.

During a median follow-up period of 42 months, 165 (25.82\%) patients experienced a CVD event. Baseline characteristics of the patients with or without CVD events are presented in Table IV.Compared with the patients without CVD events, those with CVD events were significantly older $(\mathrm{P}=0.001)$, more often male $(\mathrm{P}=0.033)$, and exhibited a higher frequency of smoker status $(\mathrm{P}=0.030)$, hypertension $(\mathrm{P}=0.023)$, diabetes $(\mathrm{P}=0.006)$ and history of MI $(\mathrm{P}<0.001)$. Patients who experienced CVD events also had significantly increased levels of $\mathrm{BMI}(\mathrm{P}=0.004)$, PLR $(\mathrm{P}<0.001)$ and hs-CRP $(\mathrm{P}=0.018)$, markedly increased platelet count and TC, significantly reduced lymphocyte counts $(\mathrm{P}<0.001)$ and markedly reduced HDL-C levels when compared with the patients who did not experience such events.

PLR as an independent predictor of CVD event in CAD patients. Once adjusted for age, gender, hypertension, DM, smoking status, BMI, lymphocyte count and hs-CRP, Cox regression analysis showed that a stronger association between PLR and the incidence of CVD event was found for the higher ratio group (PLR $>171$; HR, 1.982; 95\% CI, 1.329-2.957; $\mathrm{P}=0.001$; PLR 101-171: HR, 1.481; 95\% CI, 0.973-2.254; $\mathrm{P}=0.067)$ compared with the lower PLR group (PLR $<101)$ (Table V).

Kaplan-Meier curves further demonstrated that patients with the higher PLR had a higher incidence of CVD events when compared with those in the lower PLR group (Fig. 1; $\log$ rank, 16.275, $\mathrm{P}<0.001)$. During the follow-up period, the CVD event rate in the tertile 3 group (34.27\%) was significantly higher than in the tertile $2(25.82 \%)$ and tertile 1 groups (17.37\%; P<0.001). Patients with an elevated PLR were most inclined to experience CVD events compared with patients in the lower PLR group.

\section{Discussion}

In the present study, the relationship between PLR and CAD severity and long-term outcomes of Chinese patients following PCI were evaluated. Patients with PLR $>171$ exhibited more severe coronary artery stenosis (OR, 2.393; 95\% CI, 1.394-4.108; $\mathrm{P}=0.002$ ) and worse prognoses. The present results indicated that PLR was closely associated with the severity of CAD and that high PLR was an independent predictor of future CVD events in a Chinese Han population. 
Table IV. Clinical characteristics of patients with or without CVD event at baseline.

\begin{tabular}{|c|c|c|c|}
\hline Characteristic & With CVD event $(n=165)$ & Without CVD event $(n=474)$ & P-value \\
\hline Age, year & $62.17 \pm 10.18$ & $58.67 \pm 11.58$ & 0.001 \\
\hline Male, n (\%) & $113(68.48 \%)$ & $280(59.07 \%)$ & 0.033 \\
\hline Smoking, n (\%) & $94(56.97 \%)$ & $223(47.05 \%)$ & 0.030 \\
\hline Hypertension, n (\%) & $85(51.51 \%)$ & $194(40.93 \%)$ & 0.023 \\
\hline DM, n $(\%)$ & $37(22.42 \%)$ & $62(13.08 \%)$ & 0.006 \\
\hline History of MI (\%) & $45(27.27 \%)$ & $62(13.08 \%)$ & $<0.001$ \\
\hline $\mathrm{BMI}, \mathrm{kg} / \mathrm{m}^{2}$ & $25.19 \pm 2.73$ & $24.45 \pm 2.90$ & 0.004 \\
\hline WBC, k/ul & $7.28 \pm 1.72$ & $7.39 \pm 2.08$ & 0.413 \\
\hline Lymphocyte, $\mathrm{k} / \mathrm{ul}$ & $1.60 \pm 0.76$ & $1.88 \pm 0.85$ & $<0.001$ \\
\hline $\mathrm{PLT}, \mathrm{k} / \mathrm{ul}$ & $229.48 \pm 51.77$ & $219.87 \pm 55.55$ & 0.143 \\
\hline PLR & $170.83 \pm 74.93$ & $138.04 \pm 62.73$ & $<0.001$ \\
\hline $\mathrm{TC}, \mathrm{mmol} / \mathrm{l}$ & $4.24 \pm 1.18$ & $4.05 \pm 1.04$ & 0.058 \\
\hline $\mathrm{TG}, \mathrm{mmol} / \mathrm{l}$ & $1.79 \pm 0.88$ & $1.71 \pm 0.80$ & 0.276 \\
\hline HDL-C, mmol/l & $0.98 \pm 0.22$ & $1.02 \pm 0.23$ & 0.174 \\
\hline LDL-C, mmol/1 & $2.50 \pm 0.87$ & $2.38 \pm 0.86$ & 0.135 \\
\hline Hs-CRP, mg/l & $2.70 \pm 1.45$ & $2.41 \pm 0.99$ & 0.018 \\
\hline Statin, n (\%) & $147(89.09 \%)$ & $440(92.83 \%)$ & 0.138 \\
\hline ACEI/ARB, n (\%) & $72(43.64 \%)$ & $242(51.05 \%)$ & 0.105 \\
\hline$\beta$-blocker, n (\%) & $148(89.70 \%)$ & $439(92.62 \%)$ & 0.249 \\
\hline Aspirin, n (\%) & $147(89.09 \%)$ & $438(92.41 \%)$ & 0.195 \\
\hline
\end{tabular}

Data are presented as the mean \pm standard deviation. CVD, cardiovascular disease; DM, diabetes mellitus; MI, myocardial infarction; BMI, body mass index; WBC, white blood cell count; PLT, platelet; PLR, platelet to lymphocyte ratio; TC, total cholesterol; TG, triglyceride; HDL-C, high-density lipoprotein cholesterol; LDL-C, low-density lipoprotein cholesterol; Hs-CRP, high-sensitivity c-reactive protein; ACEI, angiotensin converting enzyme inhibitor; ARB, angiotensin receptor antagonist. Significant differences $(\mathrm{P}<0.05)$ are indicated in bold.

Table V. Cox proportional hazard regression analysis for major adverse cardiovascular events.

\begin{tabular}{lcrr}
\hline Coefficients & Hazard ratio (95\% CI) & 95\% CI & P-value \\
\hline PLR $<101$ & $1.000^{\mathrm{a}}$ & - & 0.067 \\
PLR 101-171 & 1.481 & $0.973-2.254$ & $\mathbf{0 . 0 0 1}$ \\
PLR $>171$ & 1.982 & $1.329-2.957$ & $\mathbf{0 . 0 0 3}$ \\
Age (per year) & 1.022 & $1.008-1.037$ & $\mathbf{0 . 0 1 2}$ \\
HT (vs. non-HT) & 1.480 & $1.089-2.013$ & $\mathbf{0 . 0 0 2}$ \\
DM (vs. non-DM) & 1.796 & $1.241-2.598$ & $<$ \\
History of MI (vs. non-history of MI) & 2.095 & $1.483-2.961$ & $\mathbf{0 . 0 0 1}$ \\
log CRP (per log unit) & 1.154 & $1.027-1.297$ & $\mathbf{0 . 0 1 6}$
\end{tabular}

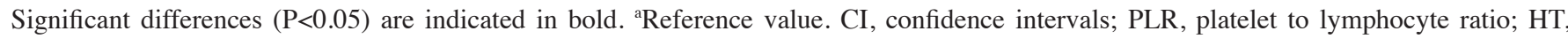
hypertension; DM, diabetes mellitus; MI, myocardial infarction; CRP, C-reactive protein.

Increased platelet counts and platelet activation have an important role in thrombus formation and the progression of atherosclerosis. It is well-known that excessive numbers of platelets, and/or normal platelets responding to abnormal vessel walls, can result in arterial thrombosis. Furthermore, increased platelets counts have been demonstrated to promote inflammation and lead to a more aggressive course of active atherosclerosis (11). The present results demonstrated that there was an increase in platelet counts with increasing numbers of coronary stenoses. A previous clinical study has determined that higher baseline platelet counts may be associated with CVD events (2). Platelet activation is also significantly associated with platelet counts (20). Inappropriate platelet activation is an important pathogenic component of thrombosis at the site of vascular injury and leads to acute coronary syndrome (21). Meanwhile, platelets can secrete various cytokines, chemokines and other inflammatory mediators, such as interleukin (IL)-1, IL-3, and IL-6, potentially contributing to 


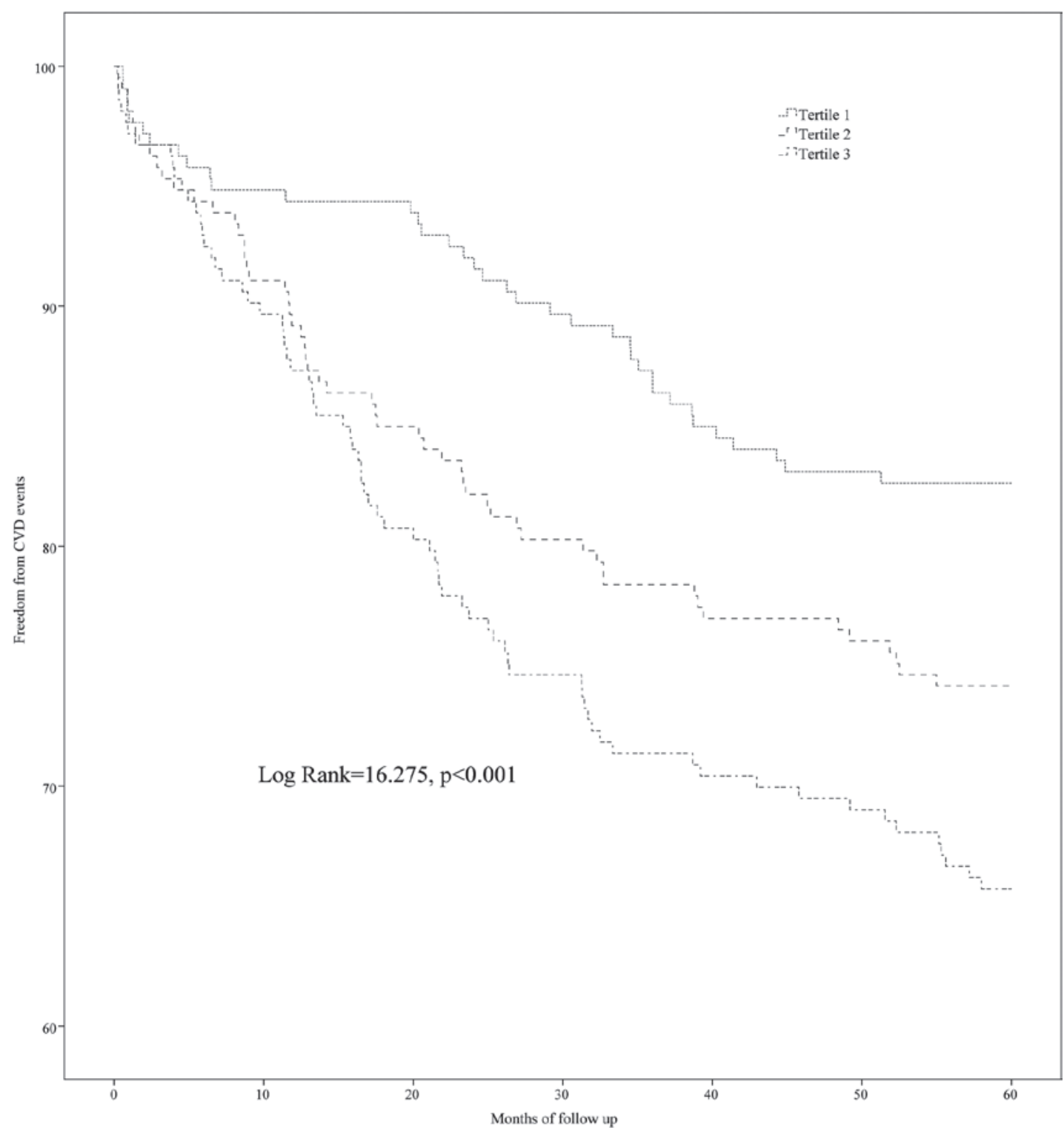

Figure 1. Kaplan-Meier survival curves based on PLR levels. Based on the tertiles of PLR levels, 639 post-percutaneous coronary intervention patients were divided into the following groups: Tertile $1(<101)$, Tertile 2 (101-171), and Tertile 3 (>171). Rate of CVD events increased with increasing of PLR (log-rank test=16.275; $\mathrm{P}<0.001)$. PLR, platelet to lymphocyte ratio.

inflammatory processes by interacting with leukocytes (22). Platelet-neutrophil binding promotes the release of vasoactive substances that locally amplify inflammatory and thrombotic responses (23). Antithrombotic activity, via the inhibition of platelet aggregation, may have novel anti-inflammatory effects (24).

Previous prospective studies have demonstrated that relative and absolute lymphocyte concentrations are lower in patients who have suffered from cardiac events $(25,26)$. The present data is in accordance with this, demonstrating that lymphocyte counts in the CVD event group were significantly lower than in the no CVD event group. A low lymphocyte count can predict the risk of reduced hemodynamics and aerobic capacity (7). Apoptosis of lymphocytes can be found in atherosclerotic lesions involved in plaque growth, lipid core development, plaque rupture and thrombosis (27). Furthermore, infiltrating lymphocytes in the ischemic and reperfused myocardium may modulate the mononuclear cell phenotype and induce tissue inhibitor of metalloproteinase-1 expression. Therefore, lymphocytes may have a key role in tissue healing (28). Similarly, lymphopenia was previously considered to be an early marker of acute myocardial infarction (29). Lymphocytopenia has also been detected in critical inflammatory states and is associated with CVD events $(6,27,30)$. Therefore, the combination of increased platelet counts and low levels of lymphocytes may provide further information as a composite marker of inflammation.

Recent studies have demonstrated that PLR is associated with a state of inflammation in patients with end-stage renal disease (31-33). For cardiovascular disease, PLR can be regarded as a novel marker for critical limb ischemia in patients with peripheral arterial occlusive disease (11), whereas PLR is considered to be a beneficial biomarker that can predict future CVD events (34). A higher PLR may reflect enhanced thrombocyte activation and a prothrombotic state (11). It has been determined that PLR is correlated with numerous 
inflammatory markers (31). The present results demonstrated that PLR is not only elevated in the multivessel disease (2- and 3 -vessel) groups compared with the 0- and 1-vessel groups, indicating that PLR may be an independent predictor of CVD events in post-PCI patients. The correlation between PLR and CAD severity was evident even after adjustment for known risk factors. These results further elucidate the role of PLR as a valuable biomarker in the progression of atherosclerosis. The present study demonstrated that PLR $>171$ identifies high-risk patients. A 56\% increased risk of major CVD events was observed in these patients, following adjustment for known cardiovascular risk factors.

There are several limitations to the present study. Firstly, results were obtained from a relatively small number of samples. Secondly, the present study followed an observational design in the Chinese population with results obtained only from single center data. Further studies with multicenter and multiracial groups are required to substantiate the present findings.

In conclusion, PLR is independently associated with CAD severity and long-term CVD events. High PLR may be a simple and easily obtainable marker of the severity of CAD and poor prognosis of post-PCI patients. Clinical use of PLR may be an effective method to identify patients who require a more aggressive therapeutic strategy to control cardiovascular risk factors.

\section{Acknowledgements}

The present study was supported by the National Basic Research Program of China (grant no. 2012CB517804) and the National Natural Science Fund for Distinguished Young Scholars (grant no. 81025002).

\section{References}

1. Libby P: Inflammation in atherosclerosis. Arterioscler Thromb Vasc Biol 32: 2045-2051, 2012.

2. Thaulow E, Erikssen J, Sandvik L, Stormorken H and Cohn PF: Blood platelet count and function are related to total and cardiovascular death in apparently healthy men. Circulation 84: 613-617, 1991.

3. Wirtz TH, Tillmann S, Strüßmann T, Kraemer S, Heemskerk JW, Grottke O, Gawaz M, von Hundelshausen P and Bernhagen J: Platelet-derived MIF: A novel platelet chemokine with distinct recruitment properties. Atherosclerosis 239: 1-10, 2015.

4. Langer HF and Gawaz M: Platelet-vessel wall interactions in atherosclerotic disease. Thromb Haemost 99: 480-486, 2008.

5. Jennings LK: Mechanisms of platelet activation: Need for new strategies to protect against platelet-mediated atherothrombosis. Thromb Haemost 102: 248-257, 2009.

6. Major AS, Fazio S and Linton MF: B-lymphocyte deficiency increases atherosclerosis in LDL receptor-null mice. Arterioscler Thromb Vasc Biol 22: 1892-1898, 2002.

7. Weng TP, Fu TC, Wang CH, Hsu CC and Wang JS: Activation of lymphocyte autophagy/apoptosis reflects haemodynamic inefficiency and functional aerobic impairment in patients with heart failure. Clin Sci (Lond) 127: 589-602, 2014.

8. Vaduganathan M, Ambrosy AP, Greene SJ, Mentz RJ, Subacius HP, Maggioni AP, Swedberg K, Nodari S, Zannad F, Konstam MA, et al: Predictive value of low relative lymphocyte count in patients hospitalized for heart failure with reduced ejection fraction: Insights from the EVEREST trial. Circ Heart Fail 5: 750-758, 2012.

9. Sunbul M, Gerin F, Durmus E, Kivrak T, Sari I, Tigen K and Cincin A: Neutrophil to lymphocyte and platelet to lymphocyte ratio in patients with dipper versus non-dipper hypertension. Clin Exp Hypertens 36: 217-221, 2014.
10. Hong LF, Li XL, Luo SH, Guo YL, Liu J, Zhu CG, Qing P, Xu RX, Wu NQ, Jiang LX, et al: Relation of leukocytes and its subsets counts with the severity of stable coronary artery disease in patients with diabetic mellitus. PLoS One 9: e90663, 2014.

11. Gary T, Pichler M, Belaj K, Hafner F, Gerger A, Froehlich H, Eller P, Rief P, Hackl G, Pilger E and Brodmann M: Platelet-to-lymphocyte ratio: A novel marker for critical limb ischemia in peripheral arterial occlusive disease patients. PLoS One 8: e67688, 2013.

12. Ozcan Cetin EH, Cetin MS, Aras D, Topaloglu S, Temizhan A, Kisacik HL and Aydogdu S: Platelet to lymphocyte ratio as a prognostic marker of in-hospital and long-term major adverse cardiovascular events in ST-segment elevation myocardial infarction. Angiology 67: 336-345, 2016.

13. Akkaya E, Gul M and Ugur M: Platelet to lymphocyte ratio: A simple and valuable prognostic marker for acute coronary syndrome. Int J Cardiol 177: 597-598, 2014.

14. Cong YL, Jin DM, Wang HL, Okada T and Peng ZH; Coordination Group for Reference Range of Venous Blood Examination in Chinese Adults: Establishing the reference range of venous blood measured by automated haematology analyzer in Chinese adults. Zhonghua Yi Xue Za Zhi 83: 1201-1205, 2003 (In Chinese)

15. Jono S, Ikari Y, Shioi A, Mori K, Miki T, Hara K and Nishizawa Y: Serum osteoprotegerin levels are associated with the presence and severity of coronary artery disease. Circulation 106: 1192-1194, 2002.

16. Arbel Y, Finkelstein A, Halkin A, Birati EY, Revivo M, Zuzut M, Shevach A, Berliner S, Herz I, Keren G and Banai S: Neutrophil/lymphocyte ratio is related to the severity of coronary artery disease and clinical outcome in patients undergoing angiography. Atherosclerosis 225: 456-460, 2012.

17. Shah A, Gnoj J and Fisher VJ: Complications of selective coronary arteriography by the Judkins technique and their prevention. Am Heart J 90: 353-359, 1975.

18. Gensini GG: A more meaningful scoring system for determining the severity of coronary heart disease. Am J Cardiol 51: 606, 1983.

19. Bairaktari E, Hatzidimou K, Tzallas C, Vini M, Katsaraki A, Tselepis A, Elisaf $M$ and Tsolas O: Estimation of LDL cholesterol based on the Friedewald formula and on apo B levels. Clin Biochem 33: 549-555, 2000.

20. Würtz M, Hvas AM, Kristensen SD and Grove EL: Platelet aggregation is dependent on platelet count in patients with coronary artery disease. Thromb Res 129: 56-61, 2012.

21. Li W, Gigante A, Perez-Perez MJ, Yue H, Hirano M, McIntyre TM and Silverstein RL: Thymidine phosphorylase participates in platelet signaling and promotes thrombosis. Circ Res 115: 997-1006, 2014.

22. Iannacone M, Sitia G, Isogawa M, Marchese P, Castro MG, Lowenstein PR, Chisari FV, Ruggeri ZM and Guidotti LG: Platelets mediate cytotoxic T lymphocyte-induced liver damage. Nat Med 11: 1167-1169, 2005.

23. Wang K, Zhou X, Zhou Z, Mal N,Fan L, Zhang M, Lincoff AM, Plow EF, Topol EJ and Penn MS: Platelet, not endothelial, P-selectin is required for neointimal formation after vascular injury. Arterioscler Thromb Vasc Biol 25: 1584-1589, 2005.

24. Alarcón M, Fuentes E, Olate N, Navarrete S, Carrasco G and Palomo I: Strawberry extract presents antiplatelet activity by inhibition of inflammatory mediator of atherosclerosis (sP-selectin, sCD40 L, RANTES and IL-1 $\beta$ ) and thrombus formation. Platelets 26: 224-229, 2015.

25. Suzuki T, Futami-Suda S, Igari Y, Watanabe K, Ouchi M, Suzuki K, Sekimizu K, Kigawa Y, Nakano H and Oba K: Low-molecular-weight lipoprotein (a) and low relative lymphocyte concentration are significant and independent risk factors for coronary heart disease in patients with type 2 diabetes mellitus: $\mathrm{Lp}$ (a) phenotype, lymphocyte, and coronary heart disease. Lipids Health Dis 12: 31, 2013.

26. Ommen SR, Gibbons RJ, Hodge DO and Thomson SP: Usefulness of the lymphocyte concentration as a prognostic marker in coronary artery disease. Am J Cardiol 79: 812-814, 1997.

27. Núñez J, Miñana G, Bodí V, Núñez E, Sanchis J, Husser O and Llàcer A: Low lymphocyte count and cardiovascular diseases. Curr Med Chem 18: 3226-3233, 2011.

28. Frangogiannis NG, Smith CW and Entman ML: The inflammatory response in myocardial infarction. Cardiovasc Res 53: 31-47, 2002. 
29. Tamhane UU, Aneja S, Montgomery D, Rogers EK, Eagle KA and Gurm HS: Association between admission neutrophil to lymphocyte ratio and outcomes in patients with acute coronary syndrome. Am J Cardiol 102: 653-657, 2008.

30. Akpek M, Kaya MG, Lam YY, Sahin O, Elcik D, Celik T, Ergin A and Gibson CM: Relation of neutrophil/lymphocyte ratio to coronary flow to in-hospital major adverse cardiac events in patients with ST-elevated myocardial infarction undergoing primary coronary intervention. Am J Cardiol 110: 621-627, 2012

31. Turkmen K, Erdur FM, Ozcicek F, Ozcicek A, Akbas EM, Ozbicer A, Demirtas L, Turk $S$ and Tonbul HZ: Platelet-to-lymphocyte ratio better predicts inflammation than neutrophil-to-lymphocyte ratio in end-stage renal disease patients. Hemodial Int 17: 391-396, 2013.
32. Akboga MK, Canpolat U, Yuksel M, Yayla C, Yilmaz S, Turak O, Ozeke O, Topaloglu S and Aras D: Platelet to lymphocyte ratio as a novel indicator of inflammation is correlated with the severity of metabolic syndrome: A single center large-scale study. Platelets 27: 178-183, 2016.

33. Akboga MK, Canpolat U, Yayla C, Ozcan F, Ozeke O, Topaloglu S and Aras D: Association of platelet to lymphocyte ratio with inflammation and severity of coronary atherosclerosis in patients with stable coronary artery disease. Angiology 67: 89-95, 2016.

34. Çiçek G, Açıkgoz SK, Bozbay M, Altay S, Uğur M, Uluganyan $\mathrm{M}$ and Uyarel $\mathrm{H}$ : Neutrophil-lymphocyte ratio and platelet-lymphocyte ratio combination can predict prognosis in patients with st-segment elevation myocardial infarction undergoing primary percutaneous coronary intervention. Angiology 66: 441-447, 2015 . 